\title{
PREPARATION OF THE HIPPARCOS INPUT CATALOGUE. \\ ASTROMETRIC PROGRAMS FOR HIPPARCOS. PRELIMINARY ASTROMETRIC OBSERVATIONS.
}

\author{
C. Turon-Lacarrieu* and Y. Réquième** \\ * Observatoire de Paris-Meudon (France) \\ ** Observatoire de Bordeaux (France)
}

ABSTRACT.

The various aspects of the preparation of the Hipparcos Input Catalogue are presented here. The astrometric parts of the work are pointed out in more details, especially the new observations and measurements already undertaken. The astrometric proposals submitted for observation with Hipparcos are also presented, as they will allow the link between Hipparcos, Space Telescope and ground-based astrometric programs.

\section{1 - INTRODUCTION.}

The measurement principle of the satellite, the organization of its scientific preparation, as well as the methods developed for data reduction have been presented in two preceding papers (Kovalevsky, 1986. ; Lindegren, 1986). This paper will deal with the work that is being done on the Input Catalogue which lists the 100000 stars Hipparcos will observe. The astrometric work done in preparation of the Catalogue, as well as the astrometric programs proposed for Hipparcos will be discussed in more detail. These programs will make it possible to establish a connection with the ground-based programs and with those of the Space Telescope and to link the Hipparcos catalogue to a reference system.

\section{2 - THE INPUT CATAlogue.}

Given the measurement principle (measurement of large angles between stars in both fields of view) and the fact that the satellite scans the sky continuously (without the possibility of stopping in a given direction), the catalogue of stars to be observed must be compiled and tested before the satellite is launched. To test means to simulate the entire mission. Therefore, the position and magnitude of the stars to be observed must be known, as well as the scanning law of the satellite, the ephemerides of the Earth and Moon and the observing strategy (choice criteria to be adopted given the priority, the 6015

H. K. Eichhorn and R. J. Leacock (eds.), Astrometric Techniques, 605-612.

(C) 1986 by the IAU. 
apparent magnitude and the position of the stars in the field of view). The result of the simulation will make it possible to judge the quality of the proposed catalogue with respect to its scientific objectives (number of stars properly observed, number of stars observed for too long, or not enough, depending upon their magnitude, distribution of the stars observed according to the priority set by the ESA Selection Committee) as well as with respect to the mission's capacities (area with too many or too few stars to be observed, wasted or ill-used observation time). These results will also make it possible to judge the efficiency of the observing strategy.

In order to carry out these simulations and then the mission itself, the magnitudes of the stars to be observed must be known beforehand at better than 0.5 magnitude and their positions at better than 1".5. The positions of half of them must even be known at better than $1^{\prime \prime}$ in order to reconstitute the satellite's attitude. For this it is necessary to not only make intensive use of the Data Base of the Centre de Données Stellaires (CDS, Observatoire de Strasbourg) which regroups all available information (astrometry, photometry, spectroscopy, bibliography, etc...) for more than 550000 stars, but also to organize new photometric and astrometric observations for around 20000 stars. For more details on the overall preparation of the Input Catalogue and on the Consortium which is compiling it, see Turon (1983).

ESA has received 210 observation proposals from all over the world, including nearly 800000 stars (with many redundancies !). The Input Catalogue will of course be compiled from the stars proposed, following the recommendations and priorities set by the ESA Selection Committee. However, the Catalogue must also allow for the constraints imposed by the operation of the satellite (briefly given above) and by the data reduction (for more details, see Lindegren, 1986 and Kovalevsky, 1984). It should include a "basic list" or "survey" of around 60000 stars brighter than about the magnitude 8 . In reality, the limiting magnitude of the survey should vary with the galactic latitude, because of the over-concentration of stars in the galactic plane. It will vary from around $m_{V}=7.7$ in the galactic plane to around 8.7 near the poles.

The basic list will also be particularly important for the materialization of a reference system since it will include stars whose positions and proper motions will be measured more precisely. Finally, the limiting magnitudes should be clearly defined in order to facilitate future use of the Catalogue to study our galaxy, its structure, kinematics, dynamics and evolution.

Data concerning approximately 720000 stars have been received at the Observatoire de Paris in Meudon (instead of 780000 proclaimed!). On this impressive number, 156000 were not treated for various reasons (mainly due to a lack of sufficient information from the proposers), 108000 have been sent only as a source of information on double stars. The remaining 458000 stars have been processed; more than 205000 
redundant stars have been eliminated. A list without redundancies of 185000 stars was ready in mid-November of 1983 ; the 68000 others are currently being processed.

It should be emphasized that this enormous task of cross-identification would not have been possible without the Data Base of the CDS in Strasbourg and the remarkable efficiency of its interrogation procedures.

3 - ASTROMETRIC PROGRAMS PROPOSED FOR HIPPARCOS.

The various astrometric programs can be grouped under 5 main headings :

a) Connection with the fundamental catalogues and the international reference star program.

Catalogues FK4, FK4 Supplement, N30, GC and NPZT have been proposed in their entirety as well as the IRS (International Reference Star Program).

Steps should also be taken to link the Hipparcos system to the FK5 which will probably be the best dynamic reference system possible in 1988. For this reason, all the stars from FK4 and FK4 Suppl. were included in the Input Catalogue with a high priority; and as soon as the FK5 list is available, it should be verified that the great majority of stars anticipated for the FK5 (if not all of them) are also included.

A comparison of the stars common to the reference frames made up on the one hand of Hipparcos stars and on the other of FK5 stars will make it possible to link the two frames and to determine the systematic regional differences between them (Röser, 1983).

It is also important to include the IRS stars in order to link the ground-based measurements and Hipparcos. However, all the $\sim 40000$ siars in the IRS cannot be included in the Input Catalogue, since competition with other programs is too great, especially in the magnitude 9-10 range.

b) Connection with the astrometric ground-based observation programs.

- Connection with the programs measuring proper motions with respect to galaxies (Lick, Tantenburg).

A sufficient number of stars common to Hipparcos and these programs must be provided for. 
- Connection with parallaxes measurement programs.

All the stars in the USNO program accessible to Hipparcos $\left(m_{V}<13\right)$ must be included, as well as some "standard" stars from the programs of the various observatories which measure parallaxes.

- Connection with Earth rotation programs.

Here, too, it must be verified that a sufficient number of stars from these programs are included in the Hipparcos program.

\section{c) Connection with occultation programs.}

- by the Moon,

- by Jupiter and its satellites,

- by Neptune, Uranus and Pluto,

- by the minor planets.

\section{d) Observation of minor planets.}

Sixty-two minor planets were proposed, mainly to improve the definition of the dynamic reference system, to detect their possible binarity and to determine their mass. Most of them at the moment do not have ephemerides precise enough to be directly included in the Hipparcos observing program. Preliminary observations (meridian circles and astrographs) have been engaged in order to improve foreknowledge of their orbits so as to predict their positions at different measurement epochs with Hipparcos. Moreover, their magnitudes are often not well known and are sometimes variable (body of irregular shape). Here, too, preliminary observations are necessary.

The observation of minor planets by Hipparcos should make it possible to directly link the Hipparcos catalogue to the dynamic reference system defined by the motions of the minor planets and materialized by their successive positions. Söderhjelm and Lindegren (1982) showed that the residual rotation of the Hipparcos frame could be determined in this way with a precision of about several milliarcseconds.

\section{e) Linking the Hipparcos Catalogue to an extragalactic reference system.}

In addition to linking the Hipparcos catalogue to the measurement programs of proper motions with respect to galaxies (photographic astrometry, cf. 3.b), it has also been proposed to link it to the VLBI system.

Two methods are being developed. The first, and it would seem the best, is to observe radio-stars. These can be observed both with VLBI and with Hipparcos without any other intermediary observations. Froeschlé and Kovalevsky (1982) showed that the error on determining 
the residual rotation can be expressed by the following relationship :

$$
\sigma=2.5 \varepsilon / \sqrt{\mathrm{N}}
$$

$\varepsilon$ being the error of VLBI measurements and $N$ the number of radio-stars used.

If $\varepsilon=0 " .002$ (which is not overly optimistic in light of the current progress of this technique), observing 15 stars will suffice to determine the residual rotation of the Hipparcos frame with respect to the VLBI system, with a precision of 0.001 "/year. A list of around 200 candidate stars is now being prepared : numerous radio (VLBI, VLA) and optic (meridian circles) observations. These radio-stars will be included in the Hipparcos observing program with a very high priority. (For more details, see Lestrade et al., 1986).

The second solution, which apparently yields results not nearly as precise as the first method (Froeschlé and Kovalevsky, 1982), consists in linking the quasars observed with VLBI to stars observed by Hipparcos by simultaneous observation with the Space Telescope of the Hipparcos stars and of the quasars optical counterparts. The list of quasars considered was drawn up by a working group of IAU Commission 24 (Argue et al., 1984 ; de Vegt, 1986). A list of candidate stars which satisfied the Hipparcos and the Space Telescope observing conditions has also been drawn up. These stars are currently being observed with "Speckle interferometry" in order to detect double stars. (For more details, see also Argue, 1986).

These two methods should made it possible to link the Hipparcos catalogue to the VLBI reference system with an accuracy better than 0.001 "/year (may-be even better than 0.0005 "/year ?).

4 - ASTROMETRIC OBSERVATIONS IN PROGRESS FOR THE PREPARATION OF THE INPUT CATALOGUE.

In paragraph 2, it was seen that $1^{\prime \prime} .5$ (r.m.s.) was the precision required on the positions of the stars to be measured by Hipparcos and $1^{\prime \prime}$ for more than half in order to reconstitute the satellite's attitude. In addition, in order to avoid the "grid ambiguity" (cf. Kovalevsky, 1984 or Lindegren, 1986). those responsible for the data reduction would prefer that the greatest possible number of stars from the program have positions known at better than 0".5. This makes it necessary to organize ground-based astrometric observations and measurements before Hipparcos is launched. Approximately 20 to 30000 stars must be remeasured (Réquième, 1982). Stars in the northern Hemisphere will be observed using the automatic meridian circle at the Observatoire de Bordeaux; those in the southern Hemisphere will be measured on ESO "Sky Survey" plates. 
a) First meridian circle observations (Observatoire de Bordeaux).

For more details, see Réquième (1986).

- Test program of weak stars (red dwarfs) from the Gliese Catalogue (stars closer than $22 \mathrm{pc}$ ).

This program made it possible to verify that the limiting magnitude accessible to the meridian circle and to Hipparcos were almost identical. 379 stars brighter than $\mathrm{m}_{\mathrm{V}}=12.5$ having declinations $\mathrm{b}$ ctween $-17^{\circ}$ and $+80^{\circ}$ have been observed.

- Radio-star program.

205 radio-stars which were candidates to establish the Hipparcos VLBI link (cf. 3.e) are being observed using the meridian circle at the Observatoire de Bordeaux. The problem is complicated by the fact that many of them are large amplitude variable stars.

- Minor planet program.

42 of the 62 minor planets proposed for Hipparcos have already been observed at least twice using the meridian circle at the Observatoire de Bordeaux. An intensive observation campaign was carried out on Massalia in October 1983 for comparison with ephemerides and improvement the computing procedures.

The minor planets which pass the furthest south should be observed photographically.

\section{b) Plate measurements. First tests.}

Measurements will begin only after the first version of the Input Catalogue is completed in order to avoid having to redo them several times successively on the same plate (placing the plate and measuring the reference stars make up an important part of the total measuring time).

Therefore, only preliminary tests have been carried out :

- tests of the various measuring machines involved : measurements of copies of the same ESO plate;

- comparison of the various reduction programs;

- comparison of the original plate and two copies at Garching ;

- testing of the precision attainable for position measurements on Schmidt plates : the precision has been improved by a factor 2 (0".30 to $0^{\prime \prime .15)}$ when the reference stars used are those measured beforehand by the meridian circle rather than AGK3R stars. 
5 - CONCLUSION.

The many interactions and the amount of collaboration necessary and achieved on the one hand between the two spatial astrometric projects (Hipparcos and the Space Telescope Astrometry FGS ) and on the other hand between both of these and the astrometric ground-based programs must be pointed out. In addition, numerous statistical programs were proposed for Hipparcos which could not be entirely satisfied, given the number of proposals and the observation constraints imposed on the Hipparcos program. These programs may be excellent candidates for the ground-based instruments and in particular for the automatic meridian circles.

\section{REFERENCES.}

- Books :

- "Hipparcos, Report on Phase A Study", 1979, edited by ESA, ESA-SCI (79), 10, Dec. 79.

- "The scientific aspects of the Hipparcos space astrometry mission", 1982, Strasbourg (France) Colloquium, 22-23 Feb. 82, ESA-SP-177.

\section{- Papers :}

- Argue, A.N. et al., 1984 , Astron. Astrophys. to be published.

- Argue, A.N., 1986, IAU Symp. Nr 109, to be published. (*)

- De Vegt, C. 1986, IAU Symp. Nr 109, to be published.

- Froeschlé, M. and Kovalevsky, J., 1982, Astron. Astrophys. 116, 89.

- Kovalevsky, J., 1984, "Prospects for Space Astronometry", Space Science Review, Vol. 39, p. 1.

- Kovalevsky, J., 1986, IAU Symp. Nr 109, to be published.

- Lestrade, J.F., Preston, R.A., Neill, A.E., Mutual, R.L. and Phillips, R.B., 1986, IAU Symp. Nr 109, to be published.

- Lindegren, L., 1986, IAU Symp. Nr 109, to be published.

- Réquième, Y., 1982, Strasbourg Colloquium, op. cit.

- Réquième, Y., 1986, IAU Symp. Nr 109, to be published.

- Röser, S., 1983, "FAST Thinkshop", Asiago (Italy) 24-27 May 83, edited by P.L. Bernacca, University of Padova (Italy).

- Söderhjelm, S. and Lindegren, L., 1982, Astron. Astrophys. 110, 156.

- Turon, C., 1983, IAU Coll. Nr 76, Middletown (USA).

(*) IAU Symp. $\mathrm{Nr} 109$, "Astrometric Techniques", Gainesville (Florida, USA), 9-12 Jan. 1984 . 
Discussion:

COLE:

What considerations for spectral type are you making in selecting stars for the HIPPARCOS input catalogue?

TURON:

We are only supposed to verify that there are enough stars in each catagory, so that absolute magnitude calibraton will be feasible. We are bound by the recommendations of the ESA Selection Committee. We must make sure that important groups are considered without trying to achieve a representative sample of the stars.

SMITH: TURON:

HQG: What are the rejection criteria for the ILS stars?

Up to now we have included all of them.

Maybe you and your colleagues are too accomodating. I suggest you reject all requests for the inclusion of stars for which there is not photo-identification.

van ALTENA:

(laughter)

shorter exposures. We really don't need $20^{\mathrm{m}}$ stars.

WALTER:

What is the deadline for including the latest values of parallaxes and radial velocities in the Input Catalogue so that the HIPPARCOS data reduction can use them if desired?

TURON:

All parameters necessary for the operation of the satellite will be available, and thev will be included in the catalogue. 